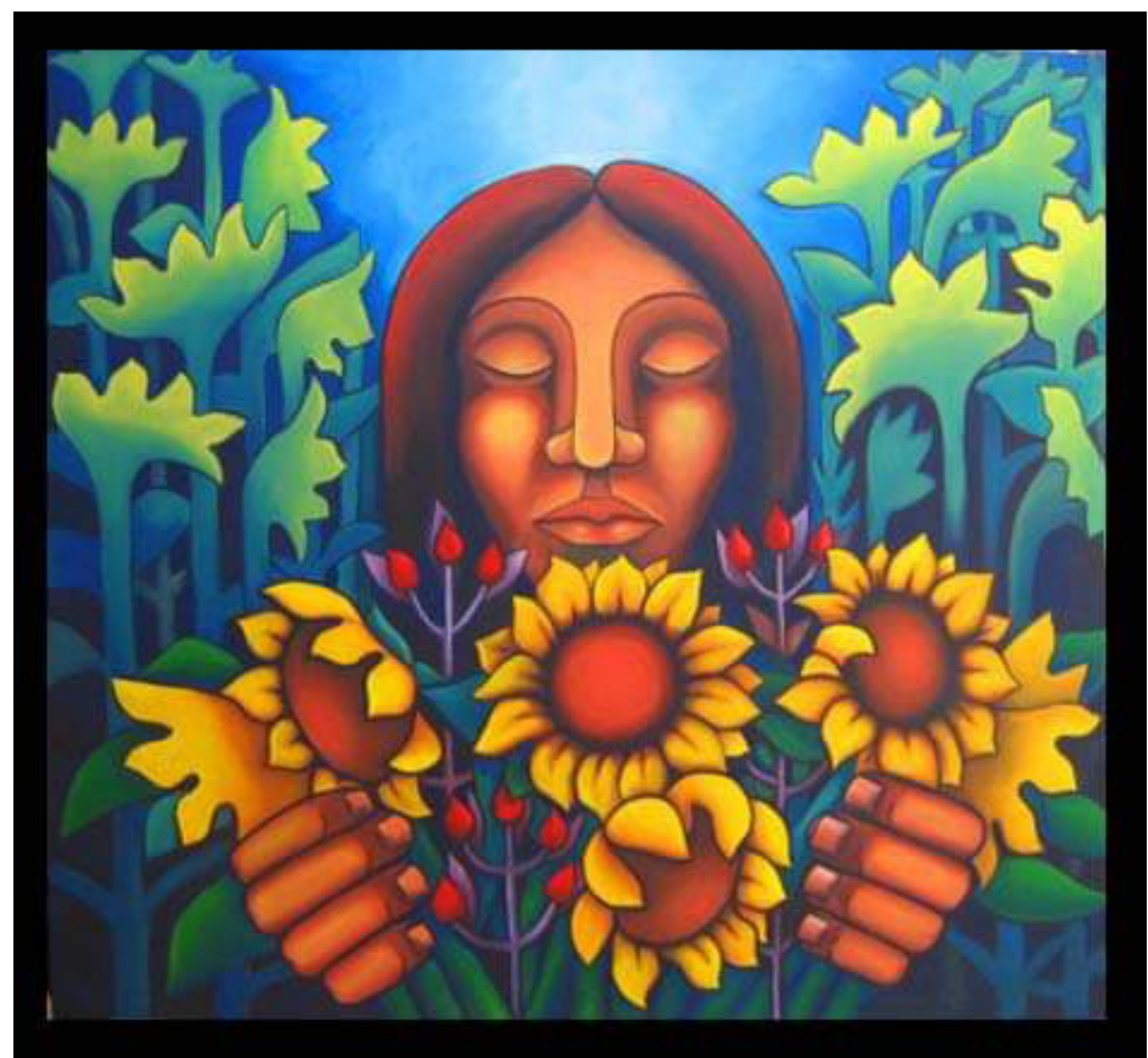

Artista: Heri Tapia 


\title{
Formación de maestros(as) en América Latina: algunos problemas y perspectivas para este tiempo
}

\author{
TEACHER TRAINING IN LATIN AMERICA: SOME PROBLEMS AND PERSPECTIVES FOR OUR TIME
}

FORMAÇÃO DE PROFESSORES (AS) NA AMÉRICA LATINA: ALGUMAS QUESTÕES E PERSPECTIVAS PARA ESSE TEMPO

\section{Alejandra Birgin 2/ alebirgin@gmail.com}

\section{Resumen}

Esta ponencia se propone debatir acerca de la formación docente en relación con ciertas coordenadas de esta época en América Latina que atraviesan de modo específico tanto a las escuelas como a la posición docente. En esta presentación se priorizan tres problemas que caracterizan algunos de los desafíos de la formación docente en este tiempo: el primero tiene que ver con la relación entre la ampliación de la agenda de derechos (obligatoriedad, interculturalidad y memoria) y la formación docente. El segundo problema toma los discursos públicos que se producen y circulan acerca de quienes enseñarán a nuestros alumnos/as (es decir, sobre aquellos que en el presente estudian para docentes). En tercer lugar, nos detendremos en una temática central en la revisión de la agenda de la formación docente en la actualidad: la cuestión del lugar de lo regional como problema pedagógico y político. De esta forma, las tres problemáticas señalan nuevas cuestiones que se plantean en el campo de las políticas de formación docente, $\mathrm{y}$, a la vez, dan a ver la necesidad de pensar nuevas pedagogías.

\begin{abstract}
This paper discusses the training of teachers, taking into consideration certain contemporary social features that determine both school and teaching positions in Latin America. In order to describe the contemporary challenges of teacher training, three problems are highlighted: the first one is related to the expansion of the right agenda (increased number of mandatory years of education, recognition of intercultural values, and focus on memory studies) and its effects on teacher training. The second problem analyzes public discourses that describe those students who plan to become teachers. The third problem focuses on a central theme in reviewing the current teacher training agenda, i.e. the relevance of both regional and local perspectives as a political and pedagogical problem. Thus, the three problems analyzed here help to identify new issues in the field of educational policy while evidencing the importance of thinking about new pedagogies.
\end{abstract}

\section{Resumo}

Nesta apresentação se propõe debater sobre a formação docente em relação a certas coordenadas contemporâneas na América Latina que atravessam de forma específica tanto as escolas como a posição docente. Nesta apresentação, priorizam-se três problemas que caracterizam alguns dos desafios da formação docente nestes tempos: o primeiro tem a ver com a relação entre a ampliação da agenda de direitos (obrigatoriedade, interculturalidade e memória) e a formação docente. O segundo problema aborda os discursos públicos que se produzem e circulam sobre aqueles que ensinarão a nossos(as) alunos(as) (ou seja, sobre aqueles que atualmente estudam para ser docentes). Em terceiro lugar, fixaremos uma temática central na revisão da agenda da formação docente na atualidade: a questão do lugar do regional como problema pedagógico e político. Dessa forma, as três problemáticas destacam novas questões que surgem no campo das políticas de formação docente e, ao mesmo tempo, visualizam a necessidade de pensar novas pedagogias.

Fecha de recepción: 08 de agosto de 2015 / Fecha de aprobación: 05 de septiembre de 2015

1 A lo largo de esta ponencia se utilizarán como sinónimos docentes, profesores y maestros. Aunque tienen definiciones diversas en cuanto a niveles y generalidad en distintos países de nuestra región, aqui son considerados como intercambiables.

2 Docente e investigadora de UBA (Universidad de Buenos Aires) y UNIPE (Universidad Pedagógica), Argentina.
Palabras clave

Formación docente, políticas docentes, nuevos profesores, ampliación de derechos

Key words

Teacher training, new teachers, education policy, expansion of rights

Palavras chave:

Formação docente, políticas docentes, novos professores, ampliação de direitos 
Debatir acerca de la formación docente hoy nos exige, a la vez que trabajar sobre sus tradiciones, situarla en los inquietantes interrogantes de esta época que, sin duda, atraviesan de modo específico tanto a las escuelas como a la posición docente.

Partimos de la convicción de que hay, en este escenario, un espacio muy potente para las universidades pedagógicas, las facultades de educación y el conjunto de las instituciones que forman docentes en América Latina hoy. En este sentido, este encuentro es una invitación desafiante y compleja a construir colectivamente nuevos horizontes de sentido.

América Latina vive un tiempo muy particular: El inicio del siglo XXI muestra las marcas de las guerras y las feroces dictaduras, a la vez que búsquedas políticas diversas preocupadas por construir otro tiempo y fortalecer en él a nuestra región. La creación de la Unión de Naciones Suramericanas (UNASUR), la Comunidad de Estados Latinoamericanos (CELAC), y la dinamización de Mercosur, dan cuenta de una voluntad política no exenta de dificultades y disputas. Allí, en esas coordenadas, quisiera colocar y valorar este evento.

Nuestra región fue laboratorio de las políticas neoliberales desde hace casi tres décadas, y nuestros sistemas educativos están atravesados por esa impronta. Algunos países (no sin conflicto interno) abonan dichas perspectivas (México, Colombia, Perú) y otros se mueven más en lo que Sader (2009) denomina una construcción alternativa posneoliberal que abarca experiencias de diversas intensidades en el marco de aquello que (retomando a Gramsci) no termina de morir ni termina de nacer. En todo caso, si hubiera que señalar rasgos comunes en todas esas experiencias de construcción alternativa es la lucha por la hegemonía, que se hace más nítida cuando se trata de la expansión de los derechos, de una reivindicación profunda de la construcción regional y, sobre todo, de una revitalización de los Estados. Se entiende así que la cosa pública y el bien común se defienden por medio del Estado, que es condición y garante a la vez. Por supuesto, las diferencias son múltiples 3 .

3 Solo como ejemplo, no es lo mismo el Estado en la perspectiva que consagra la nueva Constitución boliviana -un estado multinacionalque en el caso de Uruguay, que es un Estado unitario.
Ahora bien, ¿qué lugar le cabe a lo educativo, en particular a la formación docente en estas disputas contemporáneas? Philippe Meirieu dice algo muy interesante: la formación de los docentes es una problemática relativamente nueva en términos históricos. Mientras la educación era de y para las elites, funcionaba por complicidad cultural. Pero lo que renueva radicalmente la formación de los docentes es la democratización del sistema educativo, es el deseo de una sociedad de no dejar que el saber quede reservado a una pequeña elite, sino que sea accesible a todos (Birgin, 2012).

Se trata de un problema de la mayor importancia ética, política, teórica y práctica que está, me parece, en el corazón de las preguntas contemporáneas que compartimos aquí. ¿Cómo se posiciona/interviene la formación docente ante estos desafíos? ¿En qué condiciones institucionales? ¿Qué nuevos problemas se plantean? ¿Qué relación establece con la producción de nuevas pedagogías?

Intentar responder esas preguntas exige una salvaguarda: muchas veces el discurso más escuchado acerca de la formación docente la plantea como una varita mágica que logrará resolver las cuestiones más simples y también las más difíciles a las que se encuentran enfrentadas la sociedad y las escuelas. Pareciera además que cuanto más se complejiza este tiempo, más se alude a la formación de los docentes que están trabajando y a las escuelas como "solución"4.

Así, alrededor de la formación se ha armado un argumento en cascada que dice: el mundo cambia, las enseñanzas que deben transmitir las escuelas son cada vez más complejas, la formación recibida por los docentes es (al menos) insuficiente. Entonces, la capacitación del docente aparece como la via reggia para atender todas y cada una de esas problemáticas. Como señala A. Boissinnot (2010), la formación docente es motivo de debate y de revisión cada vez que los resultados del sistema educativo se muestran insuficientes.

Nos inquieta una definición inflacionada 5 de la potencia de la formación donde operan capas más o menos

4 Hemos desarrollado este punto en: Más allá de la capacitación. Debates acerca de la formación de los docentes en ejercicio (Birgin, 2012).

5 Tomamos aquí prestada la expresión de Marie Duru-Bellat (2006) en L'inflation scolaire. Les désillusions de la méritocratie. Allí sostiene la necesidad de interrogarse sobre las derivas y efectos perversos posibles 
sedimentadas, fantasmas, discursos y prácticas múltiples que se fueron superponiendo entre las experiencias anteriores y la nuestra. Sabemos que la formación docente es parte de un complejo entramado de políticas y tradiciones docentes. ${ }^{6}$

Si algo caracteriza a este tiempo es que conviven en él distintas intensidades y ritmos en simultáneo en diferentes esferas sociales (lo nuevo y lo viejo, lo que está por nacer). Se trata entonces de nuevas y viejas dinámicas entre las instituciones y las políticas, entre los individuos y los procesos más amplios. Se trata del interjuego entre las dimensiones subjetivas y objetivas del tiempo. Hace falta entonces atender a los préstamos y reconfiguraciones que se dan en los cambios sociales que no siguen, precisamente, una línea recta (Dussel, en prensa).

Nos parece que, en ese sentido, vale la pena leer las transformaciones contemporáneas y pensar nuestra intervención en ellas desde la formación docente: no hay procesos puros ni lineales, sino capas superpuestas y variaciones ligadas a contextos y tradiciones específicas. Los acuerdos son, entonces, históricos y contingentes.

En esta presentación prioricé tres problemas que caracterizan algunos de los desafíos de este tiempo vinculados con la formación docente: el primero tiene que ver con la relación entre la ampliación de la agenda de derechos (obligatoriedad, interculturalidad, paz y memoria) y la formación docente. El segundo con la pregunta acerca de quién enseñará a nuestros estudiantes (ligado a los discursos públicos acerca de los que estudian para docentes). En tercer lugar, me voy a detener en una temática que considero central en la revisión de la agenda de la formación docente en la actualidad: la cuestión del lugar de lo regional como problema pedagógico y político.

de una expansión de la escolaridad pensada solo en términos cuantitativos y que no se interroga sobre los nuevos modos de construcción de la selección escolar y social.

6 Las políticas docentes constituyen una trama que incluye la formación, la carrera, las condiciones laborales, las consideraciones públicas, la participación colectiva, entre otras. Es difícil hacer una delimitación precisa, ya que ellas son, por un lado, parte de las políticas educativas, a la vez que están interconectadas con otras, como las políticas respecto del Estado o del empleo.

\section{Relación entre la ampliación de la agenda de derechos y la formación docente}

Uno de los lugares donde se visualiza más claramente la envergadura de los cambios socio-históricos con los que convivimos son las transformaciones en las formas de producción, circulación y transmisión de los conocimientos, así como en la producción de otras/ nuevas formas de igualdad y desigualdad. Frente a ello, las escuelas, instituciones creadas en otro siglo, tienen múltiples dificultades y desafíos. Los ejemplos abundan. Déjenme mencionar solo uno: cómo permean a las escuelas secundarias sus mandatos originales e indiscutidos de formar a las elites locales (otrora motivo de orgullo) cuando hoy, en cambio, las atraviesa el mandato de la universalización de la escuela media. Como afirmaba P. Meirieu, eso cambia radicalmente la formación docente.

Los modos de pensar e intervenir en relación con la tarea de enseñar se organizan básicamente en dos grandes perspectivas. Por un lado, diversos planteos señalan al trabajo docente como un trabajo individual y evaluado por su eficacia (asimilada en los últimos tiempos en algunos países al rendimiento de los estudiantes en pruebas estandarizadas). Desde ahí se entiende que la práctica docente es modelizable, previsible, mediante una serie de competencias genéricas que pueden transmitirse y son, además, "todo terreno". Habría un cuerpo de saberes suficiente, normalizado, disponible para la formación docente. Los problemas específicos, además, son atendidos de modo literal y en una traducción directa en cursos de acción práctica. Los docentes se convierten en factor estratégico para una escuela de calidad, a la vez que responsables y culpables de aquello que no se logra. Hay aquí una concepción conservadora además de simplificadora, muy alejada de lo que, entendemos, sucede en el ámbito escolar.

Vamos a sostener otra posición, que reconoce la complejidad del trabajo de enseñar, su carácter político y su naturaleza institucional y colectiva (Terigi, 2004). Esta conceptualización es solidaria con sostener la escolaridad como un derecho, como un espacio igualitario y sistemático donde la humanidad transmite a las jóvenes 
generaciones su herencia. Por eso, es un espacio disputado, donde se indagan los conocimientos con un horizonte público. Esto interroga al trabajo docente y a los ámbitos y gramáticas en que se desarrolla.

Ahora bien, en un contexto de expansión de derechos (de clase, de género, de integración de niños con diversas dificultades, de etnia, etc.) se plantean problemáticas nuevas para las escuelas y para las y los que enseñan en ellas. Porque cuando los problemas se circunscribían a cómo se incrementaba la matrícula, las preguntas pedagógicas tenían menor volumen. Pero si de lo que se trata es de la concreción de los derechos (del derecho que le asiste a todo muchacho o muchacha a una porción de la cultura y recibida con un grado de libertad tal que le permita contrabandearla o serle infiel) entonces se abren muchos interrogantes pedagógicos. No es sólo un tema de voluntades políticas (que las hay muy distintas) sino de los límites y la insuficiencia del saber pedagógico disponible. Es que el saber pedagógico con el que contamos tiene problemas para enfrentar el trabajo de enseñar tal como se configura hoy, sobre todo al abordar alternativas con algún poder instituyente (Terigi, 2012).

Algunos países de la región muestran frente al desafío de la universalización y/o obligatoriedad de la escuela media, la producción de políticas extensivas tibias a la vez que experiencias intensivas renovadoras, portadoras de otros formatos y otras relaciones con las trayectorias reales, etc. Pero son aún micro experiencias, en algunas de las cuales participan activamente las universidades (Terigi, Briscioli, Scavino, Morrone y Toscano, 2013).

Parte de la clave está en los procesos de producción pedagógica frente a estos nuevos desafíos. Se abren múltiples interrogantes: ¿en qué ámbitos se produce saber pedagógico? ¿Cómo adquiere estatuto de saber la experiencia? (Novoa, 1995) ¿Qué reglas epistémicas convierten los conocimientos de la práctica en teoría publica? ¿Quién tiene legitimidad para producir saber pedagógico? ¿Qué lugar le cabe a las universidades pedagógicas? Hay allí todo un programa de investigaciones posible y necesario ligado a las nuevas pedagogías que exigen los nuevos derechos (algunas que ya se producen en las escuelas, muchas por inventar).
¿Pueden quedar afuera las instituciones que forman docentes?

En el fondo, un debate crucial para estos tiempos se produce alrededor de la pregunta acerca de si masividad y calidad de la educación pueden ir juntas. Nosotros diríamos que no puede ser de calidad una escuela si no es masiva, que no es buena si no es para todos. Por supuesto que hay una división social y cultural injusta que es resultado de nuestra historia, causa de un reparto desigual de bienes, saberes y experiencias de conocimiento. No solo eso: también esa desigualdad está ligada a qué saberes se jerarquizan y se reconocen como pertinentes (sabemos de las disputas que marcan qué saberes se reconocen como legítimos y cuáles no) y qué lugar ocupan, por ejemplo, las culturas de origen.

Los desafíos están ligados, por un lado, a los recursos específicos (Ilámense herramientas, competencias, estrategias, etc., es otra discusión) necesarios para que todos puedan aprender. Por el otro, a la afirmación de la convicción de que todo alumno es educable, de que el motor de nuestra actividad debe ser la hipótesis de la confianza en que todos pueden aprender, que las inteligencias de partida son iguales.

Sintetizando este primer punto: frente a una impronta democratizadora, saberes pedagógicos insuficientes. Escuelas que ensayan, un espacio privilegiado para las instituciones que forman docentes. Ni las prácticas escolares aisladas ni las investigaciones universitarias taxativas y alejadas de las escuelas pueden albergar respuestas. Se trata de construcciones colectivas, investigaciones novedosas, preguntas sensibles políticamente para construir nuevas pedagogías.

\section{¿Quiénes enseñarán a nuestros estudiantes? La reconfiguración institucional contemporánea de la formación de profesores}

Diversos e intensos tópicos atraviesan la reconfiguración contemporánea de la institucionalidad de la formación de profesores. En primer lugar, la importancia de la expansión actual del nivel superior resulta difícil de subestimar; aparece como la fuerza principal que moldeó cambios críticos del nivel, al punto de haber operado 
como estímulo de la mayoría de sus transformaciones de las últimas décadas (Altbach, Reisberg y Rumbley, 2009), tales como el florecimiento de sistemas académicos diferenciados y el ascenso del sector privado.

América Latina acompañó este fenómeno de "masificación intensa, continua y sin antecedentes" de la educación superior en el mundo (Ezcurra, 2011; Brunner, 2012; Rinesi, 2015) con sus diversas instituciones. En ella, la expansión del nivel superior se manifiesta no solo en una creciente diversificación de la oferta universitaria y terciaria, sino también en un crecimiento matricular e institucional significativo. Este proceso no implica solo un cambio de escala, sino que produce una serie de modificaciones en el nivel mismo que requieren ser miradas en términos históricos y actuales para comprender su envergadura y sus efectos. Diversas investigaciones revelan que la ampliación de la cobertura en el nivel superior se produce junto con la persistencia de desigualdades (Buchbinder y Marquina, 2008; Duru-Bellat y Verley, 2009; Orange, 2013). Algunos autores nombran a este fenómeno como democratización segregativa (Merle, 2000), otros como inclusión excluyente o socialmente condicionada (Gentili, 2009; Ezcurra, 2011).

En ese nuevo mapa me gustaría colocar a las universidades pedagógicas. En medio del crecimiento y la diversificación, en esta década se plantea el derecho a la educación superior. Más que su existencia efectiva, se trata de una novedad que nace de la Declaración de la CRES (Conferencia Regional de Educación Superior) del IESALC (Instituto Internacional para la Educación Superior en América Latina y el Caribe) y de la Unesco reunida en Cartagena en 2008. Plantea que la educación Superior "es un bien público y social, un derecho humano universal y una responsabilidad de los Estados" (CRES, 2008). Obviamente, es una declaración y por tanto, tiene la fortaleza y la endeblez de una declaración. Pero hay que señalar que es una novedad que da cuenta de un estado de la discusión sobre esta cuestión que no habría que subestimar ni dejar de visibilizar (Rinesi, 2015).

¿Cuáles son las condiciones que permiten plantear que la educación superior es un derecho, produciendo un giro radical en su historia elitista? No es pensable la universidad como derecho sin la expansión/obligatorie- dad de media7. Por supuesto, la diversidad en la que se instala esa proclama es amplísima, lo cual se explica en dos ejemplos extremos del sur de nuestro continente: mientras en Chile el conjunto de la universidad está arancelada y es selectiva, en Argentina la universidad estatal es de ingreso libre y gratuito ${ }^{8}$.

Las instituciones que forman para la enseñanza en la región muestran la incorporación de jóvenes de sectores sociales anteriormente excluidos del nivel de enseñanza superior $y$, específicamente, del trabajo docente. Ingresan así a los estudios para la docencia, sectores que constituyen la primera generación de la familia que llega al nivel medio y/o superior (Birgin, 2000; Pineau y Birgin, 2007; Gluz, 2011; Charovsky, 2013). Una problemática novedosa en la investigación educativa acerca de la formación docente es la inclusión de estos "nuevos" sujetos (con trayectorias educativas y pertenencias sociales diversas) que buscan formarse para trabajar como docentes. Como veremos enseguida, diferentes organismos Internacionales han contribuido a colocar esta cuestión como una fuerte preocupación en la agenda pública. Ejemplos de cómo lo plantean a lo largo de las últimas dos décadas son las citas siguientes de documentos de PREAL y el Banco Mundial:

Los bajos salarios y las condiciones precarias han empeorado particularmente el reclutamiento de nuevos profesores. La investigación reciente sugiere que aquellos que entran a programas de adiestramiento docente tienen desempeños académicos desproporcionadamente bajos. (...). En Colombia los graduados de "escuelas normalistas" (fuentes principales de entrenamiento para docentes de primaria y secundaria) obtienen los puntajes más bajos en los exámenes nacionales para entrar a la universidad (Aldana y Orozco, 1995, p. 161).

Se ha creado un círculo vicioso en el cual unos pocos graduados de secundaria talentosos aspiran a la profesión docente $y$, sin embargo, sus cupos

7 Véase al respecto los cambios en las últimas décadas en la región en Perfiles de Países del SITEAL en base a Encuestas de Hogares. Recuperado de http://www.siteal.iipe-oei.org/perfiles paises

8 En Argentina, esto se consolida en octubre de 2015, cuando es modificada la Ley de Educación Superior, estableciéndose la gratuidad y el ingreso irrestricto para el conjunto de las instituciones del nivel. 
son llenados por aquellos que no pueden postular a posiciones más prestigiosas. (Puryear, 1997, p. 14)

Para mejorar la calidad de los profesores, se deben afrontar tres desafíos básicos: reclutar, desarrollar y motivar mejores profesores. De estos desafíos, es probable que el reclutamiento (elevar el nivel de los profesores al momento del reclutamiento) sea lo más complicado para los países de América Latina y el Caribe, porque requiere que se intensifique la selectividad de la enseñanza como profesión. (Bruns y Luque, 2014, p. 23)

En los textos transcriptos hay una construcción específica de los "problemas" que conlleva la diversificación de la matrícula de formación docente. Se señala como "solución" el reclutamiento de los más talentosos. Así, la retórica del talento es convocada frente al efecto democratizador de la masificación del nivel medio y superior. Se trata de una retórica que podemos rotular como hipócrita, ya que, en el marco de la expansión de la cobertura de los distintos niveles educativos (y de la ampliación de derechos), cada vez hacen falta más docentes. Es decir, aun aceptando que debamos seleccionar a los mejores para trabajar como docentes, ¿cómo se cubriría la creciente demanda de profesores y maestros?

En el fondo, como dice Ranciere, "no es la ignorancia el problema, es el desprecio" (Ranciere, 2007, p. 130). Es el discurso que inhabilita a ocupar esos lugares, en este caso el de estudiantes de maestros, por las trayectorias y las inserciones sociales, económicas y culturales que traen ${ }^{9}$.

Ahora bien, para la formación docente y particularmente para la posición docente, la relación con los saberes y las culturas ocupa un lugar clave, estrechamente vinculado con los procesos de autorización/desautorización cultural, ya que el trabajo de enseñar es, justamente, un trabajo y un asunto público ligado a la recreación y transmisión de una porción de la cultura.

9 En los países de la región se evidencia una fuerte presencia de discursos que caracterizan a los docentes como deficitarios, en función de sus presuntas carencias y de sus imposibilidades para trabajar con problemas pedagógicos actuales. La construcción de un discurso que posiciona a los docentes en un lugar de no saber, de debilidad o de imposibilidad pedagógica, tiene consecuencias en los modos como se configura públicamente el valor de la tarea docente y sus contribuciones a la educación de las nuevas generaciones (Birgin, 2000).
Con la fuerte ampliación de la matrícula de los niveles medio y superior, se afirma que acceden particularmente a las instituciones que forman profesores sujetos con otras trayectorias formativas. Se trata de una población estudiantil heterogénea, que ha realizado distintos recorridos, con diferentes capitales culturales y otras relaciones (más o menos estrechas) con diversas culturas de origen, alejadas del canon que las instituciones formadoras y el sistema escolar han sostenido históricamente en relación con la escuela media.

La ampliación matricular e institucional de la formación de profesores -la que conlleva en muchos países una reconfiguración del sistema formador-pone en tensión los procesos de autorización cultural y la jerarquía de saberes que eran hegemónicos para la posición de profesor. Desde esa perspectiva, sostenemos que existiría una estrecha relación entre la ampliación y heterogeneización de la matrícula de formación docente, las construcciones discursivas que describen a estos estudiantes y los procesos de autorización pública de su posición docente.

En ese sentido, cuando algunos discursos señalan a los nuevos estudiantes como portadores de déficit de saberes y conocimientos y pregonan selectividad, en ese acto están, además, impotentizando a la institución de formación. ¿Qué significaría aceptar la afirmación corriente que sostiene que estos jóvenes no podrán ser buenos docentes? Por un lado, la denegación de un derecho. Pero por otro lado, significaría sostener que una institución educativa formadora de docentes no puede educar. En otras palabras, una paradoja que impotentiza a la formación misma. Para otros discursos, en cambio, el ingreso de esos nuevos estudiantes es la oportunidad de poner en discusión la jerarquía de saberes de la formación docente, así como de reconocer otras formas de producción y circulación de conocimientos que se alejan del canon establecido.

De lo que no hay dudas es de que necesitamos proponer y llevar a cabo intervenciones que, fieles a la ampliación del derecho que asiste a todo ciudadano de recibir una educación pública y ejercer la tarea de enseñar, produzcan nuevas pedagogías al alcance de los desafíos de su formación. 


\section{Aportes para una agenda regional de la formación docente}

Analizar una agenda de la formación permite ver las tradiciones en las que se inscribe, qué cuestiones se incluyen, cuáles se dejan afuera, qué jerarquías de saberes se establecen en la selección cultural y pedagógica que proponen. Toda agenda es contingente, da cuenta de disputas y preocupaciones, registra diferentes sensibilidades a los cambios contemporáneos, diferentes posiciones respecto de los nuevos derechos, etc.

En este tiempo, disputando justamente con improntas tecnocráticas y/o academicistas (Davini, 1995), parece oportuno interrogarse sobre la presencia de algunos tópicos que tienen una inclusión heterogénea en las agendas vigentes, tales como la politización del debate escolar y la cuestión de lo regional (entendiendo por tal lo latinoamericano).

Por un lado, ¿qué entendemos por politización del debate escolar? Por supuesto que no se trata de ideologización, no se trata de partidización. Sí es hacer lugar a las preguntas más generales sobre la relación entre la tarea de enseñar y la sociedad que buscamos, sí es poner en relación la cuestión de los derechos en diferentes planos con la selección cultural que proponemos. Por eso, recortar especialmente una reflexión situada, que reconozca los rasgos de esta época y los tensione con otros horizontes, es un modo de politizar la formación. Se trata de construir un horizonte pedagógico donde las memorias y la enseñanza de la historia reciente se constituyan en movilizadoras de nuevas disposiciones ético-políticas (Herrera y Ortega, 2014)10.

Pensar la construcción de lo regional no supone un movimiento exclusivo de este tiempo ${ }^{11}$. Aun cuando en los últimos años se han puesto en marcha procesos de integración, la construcción de nuestra región en términos simbólicos ( $y$, por ende, políticos) viene de larga data. Nuestra América Latina viene siendo pensada desde hace tiempo y de diferentes modos por las intervenciones que construyeron simbólicamente este territorio, sus poblaciones, sus historias, sus proble-

10 Es imposible obviar aquí una referencia solidaria con el lugar de la docencia y el proceso de paz que se desarrolla hoy en Colombia.

11 Para el desarrollo de este punto, hemos tomado Birgin, A. (2014), Estudio sobre criterios de calidad y mejora de la formación docente del Mercosur. mas y sus horizontes de futuro. Así, tanto en diversas tradiciones de pensamiento latinoamericano que se afianzaron en la producción de respuestas a partir de lo propio, como en intervenciones de organismos internacionales que buscaron diagnósticos y proveer "soluciones" para el "desarrollo" de nuestros países, se configuran diversos sentidos que construyen a la región como tal. El escenario de esta construcción aún está atravesado por efectos de la globalización neoliberal que persisten en la región, al tiempo que en ella se desarrollan procesos democratizadores que se orientan a la restitución y ampliación de derechos sociales y educativos, como mencionamos más arriba.

La construcción de lo regional implica, entre otras cuestiones, una tarea pedagógica. En especial, porque involucra definiciones sobre qué aspectos son compartidos en un territorio determinado, qué acervos culturales son comunes y vale la pena que se transmitan a las generaciones siguientes, qué problemas y resoluciones pueden construirse desde nuestras tradiciones, y qué relaciones y dinámicas deben establecerse entre las diferencias culturales que atraviesan el espacio que habitamos. Supone, también, la producción y circulación de significados acerca de lo que es nuestra región, de lo que abarca, de sus deudas y legados, de sus utopías, de sus desigualdades, de sus posibilidades. Así, lo educativo está estrechamente vinculado con los modos en que se plantee la construcción de la región, en tanto involucra un conjunto de procesos que tienen efectos en cómo ella es pensada.

Entendemos que la formación docente constituye un lugar central para desplegar la tarea pedagógica señalada. Es allí donde futuras y futuros profesoras/es, y maestras y maestros, trabajan perspectivas acerca de cómo y qué enseñar a las nuevas generaciones, qué es una educación justa e igualitaria para los ciudadanos de nuestra región, y qué sentidos se anudan en torno de esta última.

Las investigaciones muestran que en los modos actuales de abordar la construcción de lo regional parece prevalecer una mirada donde pensar la región equivale a fortalecer la visibilización y la integración de lo ya existente. Programas de intercambio, socialización de experiencias innovadoras y "buenas prácticas", posibilidades de acreditación de diversos trayectos formativos 
en nuestros países, reconocimiento de títulos, son algunos de los planteos que parecen fundarse en una idea de lo regional como la profundización de algunas articulaciones entre las propuestas formativas que ya están en curso. Este panorama no toma a lo regional como una construcción que requiere ampliar los límites en términos simbólicos, ni se interroga por los aportes que puede hacer la formación docente para desarrollar esta tarea (Birgin, 2014).

La cuestión latinoamericana, si bien tiene importantes desarrollos de producción e investigación, está prácticamente ausente de los contenidos de la formación inicial y continua de nuestros docentes. De esta forma, es poco frecuente notar la presencia de contenidos que aporten perspectivas basadas en las pedagogías latinoamericanas, en las experiencias de educación pública y popular, teniendo en cuenta que éstas suponen alternativas a los discursos pedagógicos hegemónicos. También es poco habitual hallar en la formación contenidos que problematicen el reconocimiento del carácter multicultural y plurilinguístico de la región e incorporen una preocupación por democratizar los vínculos entre los sujetos que portan estas diferencias culturales. Hacerlo supone introducir otras coordenadas epistemológicas y políticas que discutan la universalización excluyente del conocimiento eurocéntrico, y que potencien la construcción de problemas y respuestas a partir de las tradiciones de pensamiento de nuestra región, sus historias, sus deudas y sus utopías.

Entonces, la construcción regional supone una tarea permanente de configuración de nuevas perspectivas sobre lo propio de este territorio, sobre la base de registros culturales diversos, localizaciones diferentes y experiencias colectivas distintas. La región no es solo un lugar geográfico, sino también un espacio político, cultural y pedagógico en cuya construcción la formación docente puede y debe desempeñar un papel central. En ese sentido, propiciar la construcción de lo regional desde la formación docente es también un modo de politizar el debate de la formación, en tanto permite incluir en él otros territorios, otros modos de pensar las fronteras, otras voces reconocidas y, sobre todo, ampliar la mirada sobre tradiciones a transmitir y futuros a construir.
Se trata, en definitiva, de una tarea de construcción identitaria que se potencie en nuestras diferencias y haga posible un espacio común que las convoque para tornarlo más justo e igualitario. Sin dudas, allí la formación docente, nuestras universidades pedagógicas y facultades de educación tienen mucho para hacer. Porque no queremos una formación docente ajena al tiempo que vivimos. Más aun, asumimos que la formación de los que enseñamos aporta a construirlo.

Por eso, vale la pena celebrar el encuentro, poner en común nuestras preocupaciones y nuestros sueños. Porque, como dice E. Rinesi: "Ios vivos tenemos la obligación de hacer justicia a los que todavía no nacieron (no nacieron ni fueron concebidos ni imaginados ni soñados) pero que, incluso si todavía no nacieron (o justo por eso) pueden y deben ser pensados como sujetos de un conjunto de derechos que es preciso respetar" (Rinesi, 2015, p. 45).

\section{Referencias}

Aldana, E. y Orozco L. E. (1995). Colombia: Educación para la democracia y la competencia. En Puryear, J. y Brunner, J. (Comps.), Education Equity and Economic Competitiveness in the Americas. Washington, D.C.: Organization of America States.

Altbach, P., Reisberg, L. y Rumbley, L. E. (2009). Trends in global education: Tracking an academic revolution. UNESCO-World Conference on Higher Education 2009, Paris.

Birgin, A. (2000). La docencia como trabajo: la construcción de nuevas pautas de inclusión y exclusión. En P. Gentili, y F. Gaudencio (Comps.), La ciudadanía negada. Políticas de exclusión en la educación y el trabajo (pp. 221-239). Buenos Aires: Consejo Latinoamericano de Ciencias Sociales (CLACSO).

Birgin, A. (Comp.). (2012). Más allá de la capacitación. Debates acerca de la formación de los docentes en ejercicio. Buenos Aires: Paidós.

Birgin, A. (Coord.). (2014). Estudio sobre criterios de calidad y mejora de la formación docente del Mercosur. Buenos Aires: Teseo.

Boissinot, A. (2010). La formation des maîtres: débats et perspectives. Introduction. Revue internationale d'éducation de Sèvres, (55), 27-36.

Brunner, J. J. (2012). La idea de universidad en tiempos de masificación. Revista Iberoamericana de Educación Superior, 3(7), 130-143. Recuperado de https://ries.universia.net/article/ viewFile/86/144

Bruns, B. y Luque, J. (2014). Profesores excelentes: Cómo mejorar el aprendizaje en América Latina y el Caribe, resumen. Washington: Banco Mundial. 
Buchbinder, P. y Marquina, M. (2008). Masividad, heterogeneidad y fragmentación: el sistema universitario argentino 1983-2007. Los Polvorines: UNGS.

CRES (2008). Declaración de CRES 2008. Cartagena: IESALC -UNESCO .

Charovsky, M. (2013). La fragmentación de la formación docente. Su relación con el sistema educativo. Estudio de caso en el partido de Pilar, provincia de Buenos Aires. Tesis de Maestría no publicada, Facultad de Filosofía y Letras, Universidad de Buenos Aires, Buenos Aires.

Davini, M.C. (1995). La formación docente en cuestión: política y pedagogía. Buenos Aires: Paidós.

Dubet, F. (2006). El declive de la institución. Barcelona: Gedisa.

Duru-Bellat, M. (2006). L'inflation scolaire. Les désillusions de la méritocratie. Paris: Seuil et La Repúblique des Idees.

Duru-Bellat, M. y Verley É. (2009). Les étudiants au fils du temps: contexte et repères institutionnels. En Gruel, L; Galland, O.; y Houzel, G.(Éd.), Les étudiants en France: Histoire et sociologie $d^{\prime}$ une nouvelle jeunesse (pp. 195-219). Rennes: Press Universitaires de Rennes.

Dussel, I. Las tecnologías digitales y la escuela: ¿Tsunami, revolución, o más de lo mismo? En D. Pulfer, J.C. Toscano y N. Montes (Comps.),Las tecnologías digitales en educación en Iberoamérica. Madrid: Narcea. (en prensa).

Ezcurra, A. M. (2011). Masificación y enseñanza superior: una inclusión excluyente. Algunas hipótesis y conceptos clave. En N. Fernández Lamarra y M. Costa de Paula, La democratización de la educación superior en América Latina. Límites y Posibilidades. Buenos Aires: Editorial de la Universidad Tres de Febrero.

Gentili, P. (2009). Marchas y contramarchas. El derecho a la educación y las dinámicas de exclusión incluyente en América Latina (a sesenta años de la Declaración Universal de los Derechos Humanos). Revista Iberoamericana de Educación, 49, 19-57.

Herrera, M.C. y Ortega, P. (2014). Presentación dossier: Cultura política, educación y constitución de subjetividades, Revista Pedagogía y Saberes, 40, 5-7.

Merle, P. (2000). Le concept de démocratisation de I' institution scolaire: une typologie et sa mise à l' épreuve. Population, 55(1), 15-50.
Nóvoa, A. (1995). Formação de professores e profissão docente. En A. Nóvoa (Coord.), Os professores e sua formação (pp. 13-33). Lisboa: Dom Quixote.

Orange, S. (2013). L'autre enseignement supérieur. Les BTS et la gestión des aspirations scolaires. Paris: Press Universitaires de France.

OREALC/Unesco. (2012). Antecedentes y criterios para la elaboración de políticas docentes en América Latina y el Caribe. Santiago de Chile: Unesco Santiago Editorial.

Pineau, P. y Birgin, A. (2007). Esos raros peinados nuevos: ¿qué traen los futuros docentes? En M. Feldfeber y D. Andrade Oliveira (Comps.), Políticas educativas y trabajo docente. Nuevas regulaciones ¿Nuevos sujetos? (pp. 161-180). Buenos Aires: Noveduc.

Puryear, J. (1997). La educación en América Latina: Problemas y Desafíos Santiago de Chile: PREAL.

Ranciere, J. (2007). El Maestro Ignorante: cinco lecciones sobre la emancipación intelectual. Buenos Aires: Libros del Zorzal.

Rinesi, E. (2015). Filosofia (y) política de la universidad. Los Polvorines: Universidad Nacional de General Sarmiento; Buenos Aires: IEC-CONADU (Instituto de Estudios y Capacitación Confederación Nacional de Docentes Universitarios).

Sader, E. (2009). El nuevo topo. Los caminos de la izquierda latinoamericana. Buenos Aires: Siglo XXI Editores.

Terigi, F. (2004). La enseñanza como problema político. En G. Frigerio y G. Diker (Comps.), La transmisión en las sociedades, las instituciones y los sujetos: un concepto de la educación en acción (pp. 191-202). Buenos Aires: Novedades Educativas.

Terigi, F. (2012). La enseñanza como problema en la formación en el ejercicio profesional. En A. Birgin (Comp.) Más allá de la capacitación. Debates acerca de la formación de los docentes en ejercicio (pp. 109-134). Buenos Aires: Paidós.

Terigi, F., Briscioli, B., Scavino, C., Morrone, A., y Toscano, A. G. (2013). La educación secundaria obligatoria en la Argentina: entre la expansión del modelo tradicional y las alternativas de baja escala. Revista del IICE, 33, 27-46. 